\title{
Development of a Disease Severity and Responder Index for Psoriatic Arthritis (PsA) - Report of the OMERACT 10 PsA Special Interest Group
}

\author{
LAURA C. COATES, AIZAD MUMTAZ, PHILIP S. HELLIWELL, PHILIP J. MEASE, KRISTINA CALLIS-DUFFIN, \\ GERALD G. KRUEGER, NEIL J. McHUGH, VIBEKE STRAND, DAFNA D. GLADMAN, and OLIVER FITZGERALD
}

\begin{abstract}
Work within the Group for Research and Assessment of Psoriasis and Psoriatic Arthritis (GRAPPA) to develop and validate composite disease activity measures in PsA has progressed. At the Outcome Measures in Rheumatology Clinical Trials (OMERACT) 8 meeting, a core set of domains to be assessed in randomized controlled trials (RCT) and longitudinal observational studies (LOS) of PsA was agreed upon. At OMERACT 10, work to date regarding proposed composite responder indices was presented. Five proposed composite responder definitions for PsA were reviewed and discussed including new data from the GRACE (GRAppa Composite Exercise) study. There was agreement that the work to date was promising, and that developing composite outcome measures for use in RCT and LOS was important. Further work was required, including data on followup timepoints and less common phenotypes of PsA, to ensure that all subgroups were represented within GRACE. During discussion on the concept of composite measures for PsA, based on predominant/little/no involvement in several domains (such as skin versus joints, enthesitis, dactylitis, spondyloarthritis) it was acknowledged that a simple summative score encompassing all domains of PsA would be difficult to construct psychometrically and may not be appropriate. Ideally, any composite measure should retain the ability to differentiate between activity in individual domains, such as enthesitis or skin psoriasis, so that the influence of each can be assessed independently. Further work is required within the GRACE dataset to develop an optimal composite measure for PsA. Several proposals to date have shown preliminary validity according to the OMERACT filter. (J Rheumatol 2011;38:1496-501; doi:10.3899/jrheum.110278)
\end{abstract}

Key Indexing Terms:

PSORIATIC ARTHRITIS ASSESSMENT

\section{CLINICAL OUTCOME MEASURES DISEASE ACTIVITY}

$\overline{\text { From the Section of Musculoskeletal Disease, University of Leeds, Leeds, }}$ UK; Department of Rheumatology, St. Vincent's University Hospital, Dublin, Ireland; Swedish Medical Center, University of Washington School of Medicine, Seattle, WA; Department of Dermatology, University of Utah, Salt Lake City, UT, USA; Royal National Hospital for Rheumatic Diseases and University of Bath, Bath, UK; Division of

Immunology/Rheumatology, Stanford University, CA, USA; Division of

Rheumatology, Department of Medicine, University of Toronto, Centre for Prognosis Studies in the Rheumatic Diseases, Toronto Western Hospital, Toronto, Canada; and the Conway Institute, University College Dublin, Dublin, Ireland.

L.C. Coates, MBChB MRCP, LIMM, Section of Musculoskeletal Disease, University of Leeds; A. Mumtaz, MB, MRCPI, Department of

Rheumatology, St. Vincent's University Hospital; P.S. Helliwell, MA, MD, LIMM, Section of Musculoskeletal Disease, University of Leeds; P.J.

Mease, MD, Seattle Rheumatology Associates, Director of Rheumatology Research, Swedish Medical Center, and Clinical Professor, University of Washington School of Medicine; K. Callis-Duffin, MD; G.G. Krueger, MD, Department of Dermatology, University of Utah; N.J. McHugh, MD, FRCP, FRCPath, Royal National Hospital for Rheumatic Diseases and University of Bath; V. Strand, MD, FACP, FACR, Clinical Professor, Adjunct, Division of Immunology/Rheumatology, Stanford University; D.D. Gladman, MD, FRCPC, Professor of Medicine, Division of Rheumatology, Department of Medicine, University of Toronto, Centre for Prognosis Studies in the Rheumatic Diseases, Toronto Western Hospital; O. FitzGerald, MD, FRCPI, FRCP(UK), Department of Rheumatology, St. Vincent's University Hospital and Conway Institute, University College Dublin.

Address correspondence to Prof. O. FitzGerald;

E-mail: oliver.fitzgerald@ucd.ie
Psoriatic arthritis (PsA) is a multifaceted disease with involvement in many areas, including peripheral joints, skin and nail disease, entheseal involvement, dactylitis, and axial disease. Outcomes research in PsA has always lagged behind that in rheumatoid arthritis (RA), with the lack of validated clinical outcome measures for all of the disease domains a particular difficulty. There are many different outcome measures available for each of these separate aspects of the disease, but most are borrowed from related diseases, and only some of these have been validated in PsA. Until recently, there were no composite outcome measures for PsA that take all of these disease aspects into account.

It is clear that composite measures used in RA to assess disease severity and employed in responder indices, such as the Disease Activity Score (DAS) and European League Against Rheumatism (EULAR) Response Criteria, or the American College of Rheumatology (ACR) Response Criteria, assess only joint activity and therefore do not fully represent all aspects of psoriatic disease. There is an absence of evaluation of the multiple clinical domains of PsA such as enthesitis, dactylitis, spine, and skin in these composite measures. 
Recognition of this dearth of validated clinical outcome measures in PsA led to formation of a joint Outcome Measures in Rheumatology Clinical Trials (OMERACT)/ Group for Research and Assessment of Psoriasis and Psoriatic Arthritis (GRAPPA) working group to develop the research agenda of outcome measurement in PsA. There are currently more than 300 members of GRAPPA, including rheumatologists, dermatologists, and industry and patient service league representatives, with international representation.

The first step was a workshop on outcome measures in PsA held at OMERACT 7 (Asilomar, USA, 2004). During this workshop, discussion on potential domains for inclusion in PsA clinical trials led to a research agenda to identify optimal measures for each aspect of psoriatic disease and to develop effective tools where none existed ${ }^{1}$. Significant further progress was made at the OMERACT 8 conference (Malta, 2006) where a consensus was reached on the core domain set for PsA clinical trials ${ }^{2}$, based on a series of projects conducted following OMERACT 7, including a clinician Delphi exercise and data-mining from clinical trials. Instruments to assess these domains in randomized controlled trials (RCT) have largely been derived from and validated in studies of RA and psoriasis. Although they have generally shown good reliability and discrimination capacity in PsA trials with highly effective drugs ${ }^{3}$, they have not been further validated in PsA. At OMERACT 8 , no data were available on composite measures that attempted to assess multiple domains of PsA.

GRAPPA has been actively involved since OMERACT 8 in developing tools to reliably diagnose and assess PsA, including clinical, laboratory, imaging, and tissue analysis measures of disease activity. This work is pursued in individual clinical research centers, as well as collaboratively among members of the group.

At the GRAPPA Annual Meeting in 2008 (Leeds, UK) important ground work was achieved in the development of composite clinical outcome measures for PsA. Work on different proposed indices already developed was presented and this included many of the measures discussed below. Different potential approaches were also discussed using the examples of the RA and Ankylosing Spondylitis Disease Activity Score (ASDAS) and the methodology used to develop the British Isles Lupus Assessment Group (BILAG) score in systemic lupus erythematosus (SLE). Breakout groups discussed these different options, and a large collaborative exercise was proposed to start the process of development and validation of a GRAPPA/OMERACT composite disease activity measure ${ }^{4}$.

\section{Proceedings During the Special Interest Group}

At OMERACT 10 an historical overview of measures used in PsA RCT, proposed composite clinical outcome measures, was presented with a summary arguing for the development of a comprehensive composite outcome measure in PsA. This introduction was expected to provide a forum for discussion of these proposals and an opportunity for feedback and debate, and to define issues that remain in the research agenda regarding domains and outcome measures in PsA.

\section{Background and Aims of the Special Interest Group}

Philip Mease provided an historical overview of the measures used in PsA clinical trials, composite outcome measures used to date, and summarized the argument for development of a more comprehensive composite outcome measure in PsA. He reviewed some of the recent work that had preceded the special interest group (SIG) and introduced the aims of the SIG at OMERACT 10, namely:

1. To present work to date on proposed responder indices for PsA assessment in clinical trials including preliminary validation data.

2. To provide a forum for discussion of these proposals and an opportunity for feedback and debate.

3 . To define issues that remain in the research agenda regarding domains and measures in PsA.

\section{Summary of Outcome Measures Used in PsA Clinical Trials}

A number of outcome measures used in PsA clinical trials have been shown to be accurate, reliable, and discriminative $e^{2,5,6,7,8,9,10}$ (Table 1). These include measures that have been adopted from RA and psoriasis clinical trials, as well as generic measures such as the Medical Outcome Study ShortForm 36. Recognizing the importance of enthesitis and dactylitis as clinical domains, measures for these clinical features have evolved over the past several years and are now routinely performed. Several measures of enthesitis, which assess different groups of entheseal insertion sites, are being utilized, and it is anticipated that as these are evaluated, a single measure may emerge as standard. As part of this process, sites in the appendicular skeleton are being evaluated both clinically and with ultrasound to assess criterion validity of these clinical measures. These measures and their comparative performance characteristics are demonstrated in a small study of PsA patients by Gladman, et $a l^{11}$. Because spine involvement is not universal and varies from one patient to another, axial physical examination measures have not been employed in PsA clinical trials; instead, results from ankylosing spondylitis (AS) trials, using Assessment in Spondyloarthropathies (ASAS)-developed measures, are used for evidence of effect of therapies in axial PsA. Physical examination measures of the spine are reliable in axial PsA ${ }^{12}$. Several skin measures have been used, adopted from psoriasis RCT. Interestingly, patients enrolled in PsA clinical trials often have low body surface area (BSA) involvement and thus may not be reliably evaluated with the Psoriasis Area and Severity Index (PASI) score, one of the most quantitatively rigorous, because of its poorer performance in subjects with less than 3\% BSA involvement. A "target lesion" score may be used, where one lesion is evaluated over the course of the study ${ }^{5}$. More recently, several PsA trials have incorporated a modified

Personal non-commercial use only. The Journal of Rheumatology Copyright @ 2011 . All rights reserved. 
Table 1. Key outcome measures used for all domains of psoriatic disease.

\begin{tabular}{|c|c|c|}
\hline Domains & Abbreviations & Instruments \\
\hline \multirow[t]{2}{*}{ Composite measures } & CPDAI & Composite Psoriatic Disease Activity Index \\
\hline & PASDAS & Psoriatic Arthritis Disease Activity Score \\
\hline \multirow{6}{*}{ Joint assessment } & $68 / 66 \mathrm{~T} / \mathrm{S}$ & $68 / 66$ tender/swollen joint count \\
\hline & ACR & American College of Rheumatology response criteria \\
\hline & DAS & Disease Activity Score \\
\hline & PsARC & Psoriatic Arthritis response criteria \\
\hline & PSAJAI & Psoriatic Arthritis Joint Activity Index \\
\hline & DAPSA & Disease Activity in Psoriatic Arthritis Score \\
\hline \multirow[t]{3}{*}{ Axial assessment } & BASDAI & Bath Ankylosing Spondylitis Disease Activity Index \\
\hline & BASFI & Bath Ankylosing Spondylitis Functional Index \\
\hline & BASMI & Bath Ankylosing Spondylitis Metrology Index \\
\hline \multirow[t]{3}{*}{ Skin assessment } & PASI & Psoriasis Activity and Severity Index \\
\hline & Target lesion & Target Lesion Score \\
\hline & PGA & Physician's global assessment \\
\hline Nail assessment & mNAPSI & modified Nail Psoriasis Severity index \\
\hline Pain & VAS & Pain visual analog scale \\
\hline Patient global & VAS & Visual analog scale (global, skin + joints) \\
\hline Physician global & VAS & Visual analog scale (global, skin + joints) \\
\hline \multirow[t]{4}{*}{ Function/QOL } & HAQ & Health Assessment Questionnaire \\
\hline & SF-36 & Short-Form 36 \\
\hline & PsAQoL & Psoriatic Arthritis Quality of Life Index \\
\hline & DLQI & Dermatology Life Quality Index \\
\hline \multirow[t]{4}{*}{ Fatigue } & FACIT & Functional Assessment of Chronic Illness Therapy \\
\hline & KFSS & Krupp Fatigue Severity Inventory \\
\hline & MFI & Multidimensional Fatigue Inventory \\
\hline & VAS & Fatigue visual analog scale \\
\hline \multirow[t]{6}{*}{ Enthesitis assessment } & MEI & Mander Enthesitis Index \\
\hline & MASES & Maastricht Ankylosing Spondylitis Enthesitis Score \\
\hline & LEI & Leeds Enthesitis Index \\
\hline & Major & Major Enthesitis Index \\
\hline & SPARCC & $\begin{array}{l}\text { Spondyloarthritis Research Consortium of Canada } \\
\text { Enthesitis Index }\end{array}$ \\
\hline & 4-point & $\begin{array}{l}\text { Used in IMPACT studies (bilateral Achilles tendons } \\
\text { and plantar fascia) }\end{array}$ \\
\hline \multirow[t]{2}{*}{ Dactylitis assessment } & LDI & Leeds Dactylitis Instrument \\
\hline & Present/absent & Simple count of dactylitic digits \\
\hline \multirow[t]{2}{*}{ Acute-phase reactant } & ESR & Erythrocyte sedimentation rate \\
\hline & CRP & $\mathrm{C}$-reactive protein \\
\hline
\end{tabular}

Nail Psoriasis Severity Index (mNAPSI) score for evaluation of nail response ${ }^{13}$.

In an RCT of sulfasalazine in PsA, Clegg, et al utilized a composite measure of tender and swollen joint counts along with patient and physician global assessments of disease activity ${ }^{14}$. In the original etanercept trial, this composite measure was named the Psoriatic Arthritis Response Criteria (PsARC). It was not methodologically derived and does not incorporate other features of PsA such as enthesitis, dactylitis, or skin disease. The ACR response criteria and the DAS scoring system and its associated EULAR response criteria have been successfully adopted from RA for evaluation of joints in a composite fashion. Fransen, et $a l$, analyzing two phase 2 PsA trials with anti-tumor necrosis factor (TNF) agents, demonstrated that these were reliable and discriminative measures and were slightly more so than the PsARC ${ }^{3}$.

\section{Summary of Current Proposed Composite Outcome Measures}

Gladman and colleagues have recently addressed the need for a composite outcome measure in PsA. This was done using a complex analysis of datasets from a number of RCT in PsA. Interestingly, the analysis showed that the addition of the PASI score reduced the effect of the joint count, supporting the argument that the skin should be treated as an independent domain when evaluating response to therapy in PsA. Therefore, the composite measure developed (the Psoriatic Arthritis Joint Activity Index - PsAJAI) assessed only joint disease activity ${ }^{15}$.

Recently, the Vienna group used a principal component analysis of key PsA outcomes in 105 patients to identify which outcome measures best reflected disease activity. They identified 3 principal components that reached significance: patient pain and global disease activity assessment, tender and 
swollen joint counts, and C-reactive protein (CRP). A fourth component consisted of skin assessment, but this did not reach significance. After a review of existing measures, the Disease Activity in REactive Arthritis (DAREA) score was thought to be the most relevant composite outcome that encompassed all of these principal components ${ }^{16,17}$. This group has now renamed the DAREA as the Disease Activity in Psoriatic Arthritis (DAPSA) score.

A composite measure of minimal disease activity in PsA has also been developed and validated since OMERACT 9. The result of expert consensus with input from the GRAPPA members ${ }^{18}$, the measure has since been validated using independent $\operatorname{LOS}^{19}$ and RCT datasets ${ }^{20}$. However, this measure does not provide a disease activity score, but allows assessment only of whether patients achieve the minimal disease activity state.

Work developing 2 key composite measures has been initiated and led by members of GRAPPA. FitzGerald and colleagues developed a composite outcome measure based on the GRAPPA treatment grid published by Ritchlin, et $a l^{21}$. The Composite Psoriatic Disease Activity Index (CPDAI) assigns a score of 0-3 to each of the 5 domains of PsA based on disease activity and impact of disease for this domain (Table 2). The scores are added to give a total score of $0-15$, thus providing an overall assessment of disease activity ${ }^{22}$. One concern raised during development of this measure was that patients with severe disease activity in only one domain may be disadvantaged by a relatively low total score. Two solutions have been proposed for this. The first is that anyone with a single domain scored as severe would also be classified as severe overall. The second solution was the proposal of a modified CPDAI, where the total score is divided by the number of domains involved, yielding a mean CPDAI score.

Aizad Mumtaz presented preliminary validation data for the CPDAI from LOS and from patients undergoing treatment change $^{22}$. The CPDAI score showed an excellent correlation with both patient and physician global assessments. Further, in a tree analysis, total CPDAI scores $>6$ were found to be associated with treatment change. Further validation is under way and comparison with radiographic and ultrasound outcome is planned.

\section{Comparison of CPDAI and DAPSA}

A recent analysis using the Psoriasis Randomised Etanercept Study in Subjects with Psoriatic Arthritis (PRESTA) dataset compared the performance of the CPDAI and the DAPSA/DAREA. The PRESTA RCT compared 2 doses of etanercept in a large number of patients with active psoriasis and psoriatic arthritis (50 mg qiw and $50 \mathrm{mg}$ biw) ${ }^{23}$. Interestingly, while measures of joint disease, enthesitis, and dactylitis showed similar changes in the 2 treatment groups, a superior response was seen with the higher dose for skin disease. The PRESTA study therefore provides an ideal dataset with which to assess the sensitivity of composite disease activity measures. Analyses revealed that both the CPDAI and the DAPSA showed good responsiveness to change. CPDAI but not DAPSA identified a significant difference between treatment groups that was likely driven by the differential response in skin disease $(p=0.049)$. In stepwise regression analysis, enthesitis, Health Assessment Questionnaire (HAQ), dactylitis, and Dermatology Life Quality Index (DLQI) all contributed significantly to CPDAI values at baseline. Thus, while both the DAPSA and CPDAI show responsiveness in measures of arthritis, the CPDAI has a potential advantage in that it can also reflect changes in the other domains of PsA.

\section{Preliminary Data from the GRACE Exercise}

Following the GRAPPA annual meeting in 2008 and as part of the preparation for OMERACT 10, GRAPPA initiated a project that aims to develop an inclusive composite outcome measure based on real patient data, the GRAppa Composite Exercise (GRACE). Longitudinal observational data are being collected on a large cohort of PsA patients internationally. Individual outcomes assessing disease activity in all of the domains of PsA, as well as patient-reported outcome measures, are being collected. Where no consensus has been

Table 2. Composite Psoriatic Disease Activity Index (CPDAI). Score total 0-15.

\begin{tabular}{|c|c|c|c|c|}
\hline Condition & None $(0)$ & Mild (1) & Moderate (2) & Severe (3) \\
\hline Peripheral arthritis & & $\begin{array}{l}\leq 4 \text { joints (swollen or tender); } \\
\text { normal function }(\mathrm{HAQ} \leq 0.5)^{*}\end{array}$ & $\begin{array}{l}\leq 4 \text { joints but function impaired; } \\
\text { or }>4 \text { joints, normal function }\end{array}$ & $\begin{array}{l}>4 \text { joints and function } \\
\text { impaired }\end{array}$ \\
\hline Enthesitis & & $\begin{array}{l}\leq 3 \text { sites; normal function } \\
\quad(\mathrm{HAQ} \leq 0.5)^{*}\end{array}$ & $\begin{array}{l}\leq 3 \text { sites but function impaired; } \\
\text { or }>3 \text { sites but normal function }\end{array}$ & $\begin{array}{l}>3 \text { sites and function } \\
\text { impaired }\end{array}$ \\
\hline Spinal disease & & $\begin{array}{c}\text { BASDAI } \leq 4 \text {; normal function } \\
\quad(\mathrm{ASQOL} \leq 6)\end{array}$ & $\begin{array}{l}\text { BASDAI }>4 \text { but normal function; } \\
\text { BASDAI } \leq 4 \text { but function impaired }\end{array}$ & $\begin{array}{l}\text { BASDAI }>4 \text { and function } \\
\text { impaired }\end{array}$ \\
\hline
\end{tabular}

* HAQ only counted if clinical involvement of domain (joint/enthesis/dactylitis) present. HAQ: Health Assessment Questionnaire, PASI: Psoriasis Activity and Severity Index, DLQI: Dermatology Life Quality Index, BASDAI: Bath Ankylosing Spondylitis Disease Activity Index, ASQoL: Ankylosing Spondylitis Quality of Life Index.

Personal non-commercial use only. The Journal of Rheumatology Copyright @ 2011 . All rights reserved. 
reached regarding optimal outcome measures for each component of disease, e.g., enthesitis, multiple measures are being collected to allow comparison of different indices. Patients are classified by their treating physician into 2 groups: those with active disease requiring a treatment change and patients who in the opinion of their treating physician have a low disease activity or are in remission. The 2 groups can then be compared to see where significant differences exist between them and which individual outcome measures account for this difference.

To date, baseline information on 457 patients with PsA has been collected as part of the GRACE initiative, and followup data are available on 157 patients. Analysis of the many outcome measures included in the dataset has shown a difference in all key variables for those undergoing treatment change and those not, except for the mNAPSI, Maastricht Ankylosing Spondylitis Enthesitis Score, erythrocyte sedimentation rate, and Bath Ankylosing Spondylitis Metrology Index. A principal component analysis was performed for all variables included in disease activity measures with log transformation for PASI, Leeds Enthesitis Index, dactylitis, joint counts, and CRP. Factor analysis identified 5 components that were (1) patient and physician completed measures, (2) skin activity, (3) tender joint count and enthesitis, (4) swollen joint count and dactylitis, and (5) CRP. However, with regression analysis, nearly $80 \%$ of variability (adjusted $\mathrm{R}^{2}$ ) was provided by patient global disease visual analog scale (VAS) and over $90 \%$ by just 3 VAS scores (patient global, patient skin, and physician global). Dr. Helliwell therefore proposed the Psoriatic Arthritis Disease Activity Score (PASDAS) as a composite of 3 VAS scales. This is similar to what was found in the development of the PsAJAI ${ }^{15}$.

The GRACE dataset further permitted a comparison of this proposed measure with the CPDAI, DAPSA, and DAS28: all 4 measures demonstrated adequate discrimination in terms of the construct of disease activity. Interestingly, this held true for the oligoarthritis and polyarthritis subgroups but not for those with severe skin disease (PASI > 10). In addition, all these measures showed good responsiveness in the GRACE data set. A preliminary analysis of a modular approach to disease assessment rating each domain (peripheral arthritis, skin, dactylitis, enthesitis, and spine) in terms of an intention to treat was also presented, whereby each domain would be rated from $A$ to $E$ on a 5-point scale, where E represents no involvement of that domain and A severe involvement requiring disease-modifying antirheumatic drug therapy.

The GRACE initiative has provided an excellent resource for both development and testing of PsA composite outcome measures. Evident by recent analyses, further testing, refinement, and validation are required to develop the optimal composite measure for PsA. More data from the GRACE dataset at followup timepoints will allow further assessment of responsiveness to change and aid in defining disease activity states. The dataset may also require further enriching to ensure that a wide variety of disease phenotypes are included. Initial recruitment for GRACE resulted in a cohort with low mean/median skin disease activity, and later stages of GRACE recruitment have attempted to address this with recruitment of patients with significant skin involvement.

\section{Discussion at OMERACT 10}

All participants of the SIG agreed that the work to date was promising and that plans for development of a composite responder instrument for use in RCT and LOS was appropriate. It was also agreed that no decision on the optimal outcome measure could be made without further work. Concerns were raised about relatively small numbers of patients in various subgroups of PsA collected in the GRACE study, and it was agreed that additional recruitment for underrepresented populations, such as patients with more severe skin disease, should occur. It was also acknowledged that further data were required at followup to facilitate development and testing of measures.

Concerns were raised about a composite measure that included only VAS scales, which potentially may be very subjective - although many psychometric analyses have indicated VAS scales confer better sensitivity and equivalent reliability to categorical Likert scales. Further suggestions were made on approaches to analyzing the existing dataset.

A concern regarding the construct of the GRACE study was raised - that patients were divided into low and high disease activity groups based on the physicians' treatment decisions. Although similar methodology was used in the development of the DAS for RA and AS, such an approach presents a challenge when used in PsA. The majority of patients have been recruited by rheumatologists, and therefore the decision to change treatment is likely driven by articular disease, whereas treatment decisions in other cohorts may be based on skin and nonarticular manifestations. This remains a continuing discussion between rheumatologists and dermatologists in GRAPPA - hopefully future analyses within and outside the GRACE project will address this issue.

Finally, discussions concluded regarding the concept of composite measures for PsA. Concerns were raised that PsA is a highly heterogenous condition with disease activity manifested in many domains that may be unrelated. Should a composite disease score encompassing all domains of involvement be used in PsA, or would it be more appropriate to capture individual aspects of the disease? It was agreed that at this stage we did not have the ability to use a composite measure for all disease aspects and that individual outcome measures were currently the most validated measures available. However, progress has been made to define composite scores that may be useful in the future.

More discussion is required on the optimal design of such a measure. It was acknowledged that a simple summative score encompassing all domains of PsA may be difficult to construct psychometrically and may not be appropriate, as it

Personal non-commercial use only. The Journal of Rheumatology Copyright $@$ 2011 . All rights reserved. 
would not be able to differentiate between the magnitude of activity in the different domains. Ideally, any composite measure should retain the ability to be broken down into its disparate domains, as is the case with the CPDAI, so that the effects of each of these individual aspects of the disease, and their potential for differential treatment response, can be assessed. Composite measures may also need sophisticated weighting of the various components, which would be specific to psoriatic disease and should not be "borrowed" from composite measures in other diseases, such as the RA DAS.

The final comment was that a similar session with more time for discussion was required to advance the project. The chairs of the SIG commented that progress made in the next 2 years should mean that a module update can be planned for OMERACT 11 in 2012.

\section{Conclusion}

This special interest group provided a valuable opportunity to present work to date on composite measures in PsA and to provide a forum for discussion. Further work is required within the GRACE dataset to ensure that all phenotypes of PsA are included in data collection and to aid in further development of an optimal composite measure for PsA. Several proposals to date have shown good preliminary validity according to the OMERACT filter. It is intended to further develop this work within the GRAPPA organization and GRACE study, with regular teleconference discussions, meetings adjacent to rheumatology and dermatology conferences, and the annual GRAPPA meetings. We intend to present the results of these analyses at the next OMERACT meeting in 2012 within a module update, and to publish results contributed by GRAPPA and OMERACT in the field of outcome measures in psoriatic arthritis.

\section{REFERENCES}

1. Gladman DD, Mease PJ, Krueger G, van der Heidje DM, Antoni C, Helliwell PS, et al. Outcome measures in psoriatic arthritis. J Rheumatol 2005;32:2262-9.

2. Gladman DD, Mease PJ, Strand V, Healy P, Helliwell PS, Fitzgerald $\mathrm{O}$, et al. Consensus on a core set of domains for psoriatic arthritis. J Rheumatol 2007;34:1167-70.

3. Fransen J, Antoni C, Mease PJ, Uter W, Kavanaugh A, Kalden JR, et al. Performance of response criteria for assessing peripheral arthritis in patients with psoriatic arthritis: analysis of data from randomised controlled trials of two tumour necrosis factor inhibitors. Ann Rheum Dis 2006;65:1373-8.

4. Gladman DD, Landewe R, McHugh NJ, Fitzgerald O, Thaci D, Coates L, et al. Composite measures in psoriatic arthritis: GRAPPA 2008. J Rheumatol 2010;37:453-61.

5. Feldman SR, Krueger GG. Psoriasis assessment tools in clinical trials. Ann Rheum Dis 2005;64 Suppl 2:ii65-8; discussion ii9-73.

6. Gladman DD, Helliwell P, Mease PJ, Nash P, Ritchlin C, Taylor W. Assessment of patients with psoriatic arthritis: a review of currently available measures. Arthritis Rheum 2004;50:24-35.
7. Mease PJ. Assessment tools in psoriatic arthritis. J Rheumatol 2008;35:1426-30.

8. Mease PJ, Antoni CE, Gladman DD, Taylor WJ. Psoriatic arthritis assessment tools in clinical trials. Ann Rheum Dis 2005;64 Suppl 2:ii49-54.

9. Mease PJ, Menter MA. Quality-of-life issues in psoriasis and psoriatic arthritis: outcome measures and therapies from a dermatological perspective. J Am Acad Dermatol 2006;54:685-704.

10. van der Heijde D, Sharp J, Wassenberg S, Gladman DD. Psoriatic arthritis imaging: a review of scoring methods. Ann Rheum Dis 2005;64 Suppl 2:ii61-4.

11. Gladman DD, Inman RD, Cook RJ, Maksymowych WP, Braun $\mathrm{J}$, Davis JC, et al. International spondyloarthritis interobserver reliability exercise - the INSPIRE study: II. Assessment of peripheral joints, enthesitis, and dactylitis. J Rheumatol 2007;34:1740-5.

12. Gladman DD, Inman RD, Cook RJ, van der Heijde D, Landewe $\mathrm{RB}$, Braun J, et al. International spondyloarthritis interobserver reliability exercise - the INSPIRE study: I. Assessment of spinal measures. J Rheumatol 2007;34:1733-9.

13. Cassell SE, Bieber JD, Rich P, Tutuncu ZN, Lee SJ, Kalunian KC, et al. The modified Nail Psoriasis Severity Index: validation of an instrument to assess psoriatic nail involvement in patients with psoriatic arthritis. J Rheumatol 2007;34:123-9.

14. Clegg DO, Reda DJ, Mejias E, Cannon GW, Weisman MH, Taylor $\mathrm{T}$, et al. Comparison of sulfasalazine and placebo in the treatment of psoriatic arthritis. A Department of Veterans Affairs Cooperative Study. Arthritis Rheum 1996;39:2013-20.

15. Gladman DD, Tom BD, Mease PJ, Farewell VT. Informing response criteria for psoriatic arthritis. I: Discrimination models based on data from 3 anti-tumor necrosis factor randomized studies. J Rheumatol 2010;37:1892-7.

16. Schoels M, Aletaha D, Funovits J, Kavanaugh A, Baker D, Smolen JS. Application of the DAREA/DAPSA score for assessment of disease activity in psoriatic arthritis. Ann Rheum Dis 2010;69:1441-7.

17. Nell-Duxneuner VP, Stamm TA, Machold KP, Pflugbeil S, Aletaha D, Smolen JS. Evaluation of the appropriateness of composite disease activity measures for assessment of psoriatic arthritis. Ann Rheum Dis 2010;69:546-9.

18. Coates LC, Fransen J, Helliwell PS. Defining minimal disease activity in psoriatic arthritis: a proposed objective target for treatment. Ann Rheum Dis 2010;69:48-53.

19. Coates LC, Cook R, Lee KA, Chandran V, Gladman DD. Frequency, predictors and prognosis of sustained minimal disease activity in an observational psoriatic arthritis cohort. Arthritis Care Res (Hoboken) 2010;62:970-6.

20. Coates LC, Helliwell PS. Validation of minimal disease activity for psoriatic arthritis using interventional trial data. Arthritis Care Res (Hoboken) 2010;62:965-9.

21. Ritchlin CT, Kavanaugh A, Gladman DD, Mease PJ, Helliwell P, Boehncke WH, et al. Treatment recommendations for psoriatic arthritis. Ann Rheum Dis 2009;68:1387-94.

22. Mumtaz A, Gallagher P, Kirby B, Waxman R, Coates LC, Veale JD, et al. Development of a preliminary composite disease activity index in psoriatic arthritis. Ann Rheum Dis 2011;70:272-7. Erratum in: Ann Rheum Dis 2011;70:716.

23. Sterry W, Ortonne JP, Kirkham B, Brocq O, Robertson D, Pedersen $\mathrm{RD}$, et al. Comparison of two etanercept regimens for treatment of psoriasis and psoriatic arthritis: PRESTA randomised double blind multicentre trial. BMJ 2010;340:c147. 